\title{
Anesthetic preconditioning of traumatic brain injury is ineffec- tive in a Drosophila model of obesity
}

\author{
Dena Johnson-Schlitz ${ }^{1}$, Julie A. Fischer ${ }^{1}$, Hannah J. Schiffman ${ }^{1}$, Amanda R. Scharenbrock ${ }^{1}$, Zachariah P. G. Olufs \\ 1, David A. Wassarman ${ }^{2}$, and Misha Perouansky 1,* \\ a1 Department of Anesthesiology, University of Wisconsin-Madison, Madison, WI 53792, USA \\ 2 Department of Medical Genetics, University of Wisconsin-Madison, Madison, WI 53706, USA \\ * Correspondence: mperouansky@wisc.edu
}

\begin{abstract}
We tested the hypothesis that obesity influences the pharmacodynamics of volatile general anesthetics (VGAs) by comparing effects of anesthetic exposure on mortality from traumatic brain injury (TBI) in lean and obese Drosophila melanogaster. We induced TBI with a High-Impact Trauma device. Starvation-selection over multiple generations resulted in an obese phenotype (SS flies). Fed flies served as lean controls (FC flies). Adult (1-7 day old) SS and FC flies were exposed to equianesthetic doses of isoflurane or sevoflurane either before or after TBI. The principal outcome was percent mortality 24 hours after injury, expressed as the Mortality Index at 24 hours (MI24). TBI resulted in lower $\mathrm{MI}_{24}$ in FC than in SS flies (21 (2.35) and 57.8 (2.14), respectively $n=12, \mathrm{p}=0.0001$ ). Preexposure to isoflurane or sevoflurane preconditioned FC flies to TBI reducing the risk of death to 0.53 [0.25 to 1.13] and 0.82 [0.43 to 1.58], respectively, but had no preconditioning effect in SS flies. Postexposure to isoflurane or sevoflurane increased the risk of death in SS flies. Only postexposure to isoflurane increased the risk in FC flies (1.39 [0.81 to 2.38]). Thus, obesity affects the pharmacodynamics of VGAs, thwarting the preconditioning effect of isoflurane and sevoflurane in TBI.
\end{abstract}

Keywords: Drosophila melanogaster; TBI; obesity; volatile anesthetics, isoflurane; sevoflurane; preconditioning; mortality; toxicity

\section{Introduction}

Preconditioning, i.e., the capacity of anesthetics to induce tolerance to injury when administered prior to ischemia, is a potentially valuable property of volatile general anesthetics (VGAs). Anesthetic preconditioning effectively protects the brain [1] and the heart [2] from ischemic damage. Interestingly, its effectiveness in protecting the myocardium is suppressed in rodent models of obesity [3] and diabetes [4]. Suppression may be due to a pathologic entity termed 'lipotoxic cardiomyopathy' or 'fatty heart syndrome' [5]. An analogous 'lipotoxic' or fatty brain phenotype has not yet been described, and it remains unknown whether obesity also influence anesthetic preconditioning of nervous tissue.

Experimental brain injury is always conducted with anesthetic exposure, therefore non-anesthetized control groups are practically impossible, with most experiments requiring exposure to anesthesia for technical (e.g., immobility for surgery) and/or animal welfare reasons. At the same time, VGAs profoundly influence almost all aspects of brain physiology [6-9]. Adding to the confusion, anesthetic classes have diverse effect on the brain [10], further confounding the interpretation of data from experimental brain injury models, which has been acknowledged by many research groups and may contribute to the frequent failure in translating findings from mammalian models to human patients.

Crucially, fruit flies (Drosophila melanogaster) pose limited animal welfare concerns while key characteristics of traumatic brain injury (TBI) in mammals, including temporary incapacitation (concussion), ataxia, death, neurodegeneration, and shortened lifespan [1113] as well as the behavioral effects of VGAs [14] are reliably mimicked. We found that exposure of a standard laboratory fly line $\left(w^{1118}\right)$ to the VGAs isoflurane and sevoflurane 
prior to TBI effectively preconditioned the brain, as indicated by suppression of $24 \mathrm{~h}$ mortality [15]. By contrast, exposure to isoflurane after TBI increased mortality [15]. To test the hypothesis that obesity modulates anesthetic pharmacodynamics, we inflicted TBI in a fly model of obesity acquired by starvation-selection, which mimics many of the phenotypic characteristics of obesity in mammals, including increased weight and triglyceride storage as well as behavioral, anatomical and metabolic abnormalities [16-18]. Here we show that obesity thwarts anesthetic preconditioning by isoflurane and sevoflurane in the fly TBI model. These data indicate that preconditioning with VGAs in flies is responsive to biological variables and reproduces the effect of obesity on anesthetic pharmacodynamics suggested in mammalian myocardial ischemia. While this information complicates the design of experiments that require the use of anesthetics, it can be instrumentalized to improve our understanding and treatment of TBI.

\section{Results}

The SS phenotype is gradually lost over multiple generations when flies are not subjected to starvation selection (A. Gibbs, personal communication). Therefore, experiments in Figures 1, 3 and 4 were performed on $2^{\text {nd }}$ to $4^{\text {th }}$ generation SS flies. To test whether anesthetic pharmacodynamics are maintained in flies not subjected to starvation selection, we have tested flies for up to 10 generations under culture conditions with unrestricted access to standard food. Figures 2 and 5 show changes in the SS phenotype that manifest within 10 generations.

\subsection{SS flies are heavier and at increased risk of mortality after TBI}

Under our culture conditions, SS flies developed an easily recognizable obese phenotype weighing $40-50 \%$ more than FC flies ( 1.25 vs. $0.84 \mathrm{mg}$ ), in agreement with previously reported data [16]. To investigate whether the obesity phenotype alters the risk of early mortality after TBI, we determined the $\mathrm{MI}_{24}$ of $\mathrm{FC}$ and SS flies after four strikes with the HIT device, which is described in the Materials and Methods. (Fig. 1). The $\mathrm{MI}_{24}$ of FC flies was 20.1 (2.35) $n=6, C I$ [17.6 to 22.5], close to that previously reported for $w^{1118}$ flies of the same age [15] (Fig. 1). By contrast, the $\mathrm{MI}_{24}$ of SS flies was 57.8 (2.14), n=6, CI [55.5 to 60.0], which is in the top decile of $\mathrm{MI}_{24}$ values reported from a variety of fly collections [19]. In summary, changes associated with obesity increased the relative risk of TBI-induced early death 2.85 -fold CI [1.86 to 4.37$]$ ( $p<0.0001)$.

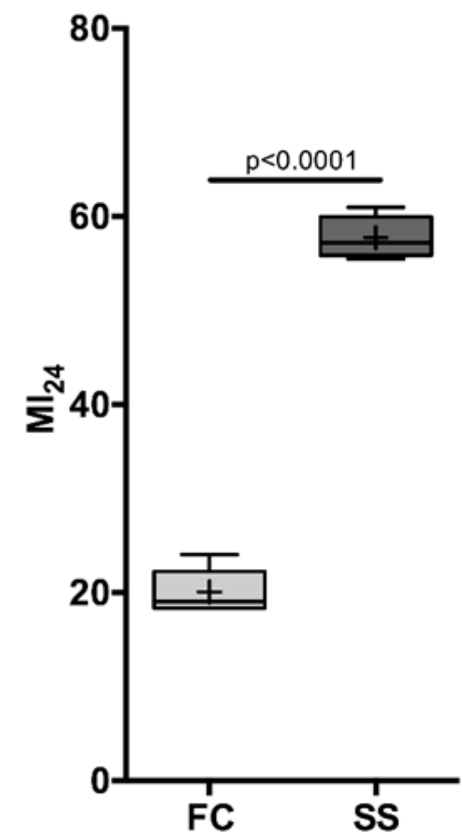


Figure 1. Obesity is associated with increased mortality. The mortality index at $24 \mathrm{~h}\left(\mathrm{MI}_{24}\right)$ after traumatic brain injury (TBI) was determined in 1-7-day-old lean FC (fed control) and obese SS (starvation-selected) flies. The relative risk of death was 2.85 [1.86 to 4.37], $n=6$ for each group. + indicates the mean, the horizontal line indicates the median, the box indicates $25^{\text {th }}$ to $75^{\text {th }}$ percentile and the whiskers extend to the minimal and maximal values.

\subsection{Early mortality is positively correlated with fly weight}

To examine whether the high $\mathrm{MI}_{24}$ of SS flies was attributable to their increased weight, we examined male flies from four additional fly lines whose weights varied over a 3-fold range from 0.5 to $1.7 \mathrm{mg}$, bracketing the weight of FC and SS flies. In addition to FC and SS flies, we included two Drosophila melanogaster lines $w^{1118} 0.55(0.02) \mathrm{mg}$ and 2P9 $0.73(0.02) \mathrm{mg}$ as well as lines of Drosophila virilis $1.57(0.03) \mathrm{mg}$ and Drosophila funebris 1.62 $(0.05) \mathrm{mg} . w^{1118}$ is a standard laboratory line and 2P9 is an uncharacterized P-element insertion line. We found that fly weight was highly correlated with the $\mathrm{MI}_{24}\left(\mathrm{R}^{2}=0.96\right)$ (Fig. 2). This might be expected because both the force imparted on the flies and the energy they were subjected to should be proportional to their mass (i.e., force $=$ mass $X$ acceleration $(F=m a)$ and energy $=1 / 2$ mass $X$ velocity of impact squared $\left.\left(E=1 / 2 \mathrm{mv}^{2}\right)\right)$. The close, but not perfect, correlation between the weight of a fly and the $\mathrm{MI}_{24}$ leaves room for other factors to affect the $\mathrm{MI}_{24}$ (e.g., genetic background). We previously found that when tested at 0-7 days old, inbred fly lines from the Drosophila Genetic Reference Panel (DGRP), whose males vary in weight from 0.58 to $0.87 \mathrm{mg}$ [20], had $\mathrm{MI}_{24}$ values that varied from 8 to 58 [19], which exceeds the expected variability based exclusively on weight (23 to 37 predicted by the data in Figure 2). These data indicate that weight plays a major role in increasing TBI-induced mortality of SS over FC flies, but it remains possible that increased mortality of SS flies results from severe secondary injuries due to cellular and molecular effects associated with obesity.

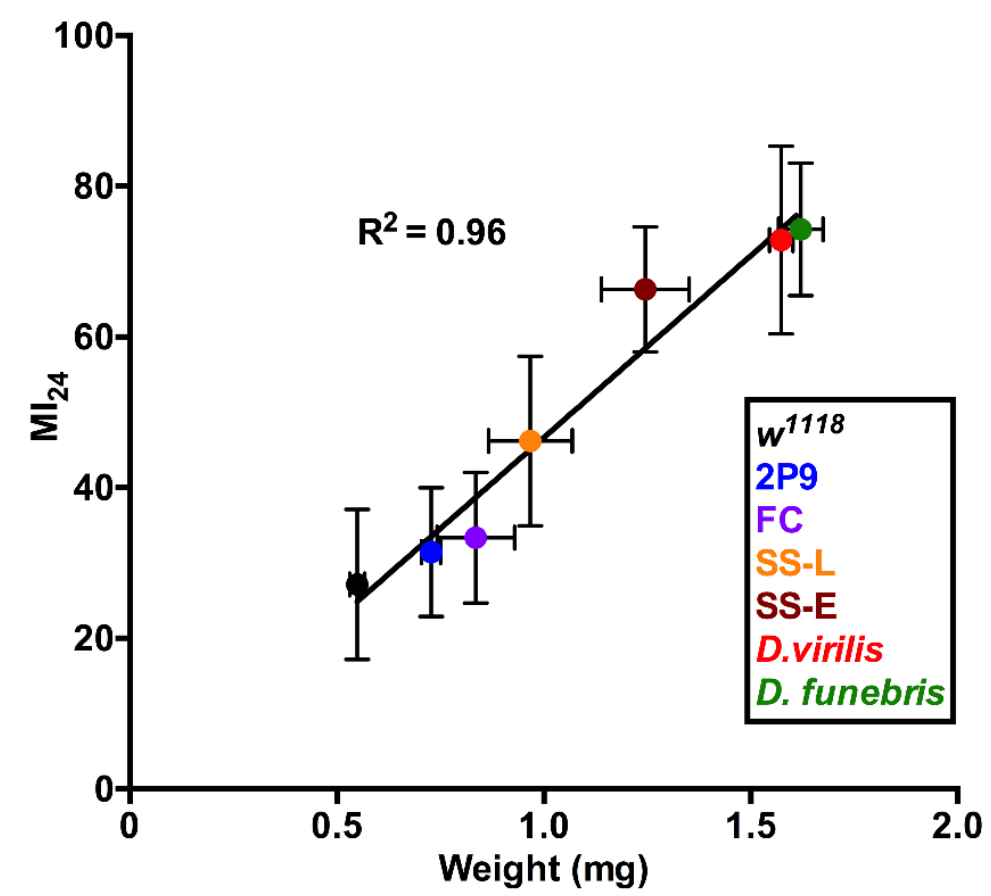

Figure 2. Early mortality $\left(\mathrm{MI}_{24}\right)$ is correlated with weight. We determined the weight (independent variable) and the $\mathrm{MI}_{24}$ in males for four lines of D. melanogaster $\left(w^{1118}, \mathrm{FC}, \mathrm{SS}, 2 \mathrm{P9}\right)$ and for $D$. funebris and D. virilis. FC flies weighed $0.84(0.09) \mathrm{mg}$. SS flies from the $2^{\text {nd }}$ to $10^{\text {th }}$ generation, i.e., early (E) generations, maintained an obese phenotype (SS-E) and weighed $1.25(0.1) \mathrm{mg}$. The late (L) generation SS flies that were losing the obese phenotype (SS-L) weighed 0.97(0.1) mg at generation 20. Fly mass was determined in males by averaging the results of 12 replicates of 30 flies on three different days for each line. All lines were tested at 1-8 days old. The data were fitted with a simple linear regression. SS flies were tested at two time points of their reproduction cycles in the lab early $\left(2^{\text {nd }}-10^{\text {th }}\right.$ generation, SS-E) and late (generation 20 , SS-L). By the $20^{\text {th }}$ generation, SS-E flies 
had partially lost the obese phenotype. For the determination of weight, $n=4$ for each datapoint. For determination of the $\mathrm{MI}_{24}, n=14$, except for SS-E where $n=24$.

\subsection{Anesthetic pretreatment does not precondition SS flies.}

To test the hypothesis that obesity influences anesthetic pharmacodynamics, we assayed the effect of anesthetic exposure prior to TBI. In FC flies, pretreatment with equianesthetic doses of isoflurane $(4 \% \mathrm{~h})$ or sevoflurane $(7 \% \mathrm{~h})$ reduced the $\mathrm{MI}_{24}$ from 17.5 (5.1, $n=24)$ to $9.4(4.1, n=16)$ and to $14.3(3.5, n=8)$, respectively (Fig. 3). Thus, exposure to either agent preconditioned flies to TBI. Preconditioning by isoflurane and sevoflurane resulted in a reduction of the relative risk of death to 0.53 [CI 0.25 to 1.13 ] and 0.82 [CI 0.43 to 1.58], respectively. By contrast, preexposure of SS flies with the same doses of isoflurane or sevoflurane did not precondition to TBI. The $\mathrm{MI}_{24}$ without preexposure was 58.1 (6.6, $n=27)$ and with preexposure was $60.7(10.9, n=21)$ and $62.8(11.4, n=8)$ for isoflurane and sevoflurane, respectively. We conclude that while the effectiveness of preconditioning in FC flies is comparable to the previously reported protective effects of these agents in $w^{1118}$ flies $[15,21]$, the obese phenotype generated by starvation-selection is associated with changes that thwart molecular mechanisms underlying anesthetic preconditioning.

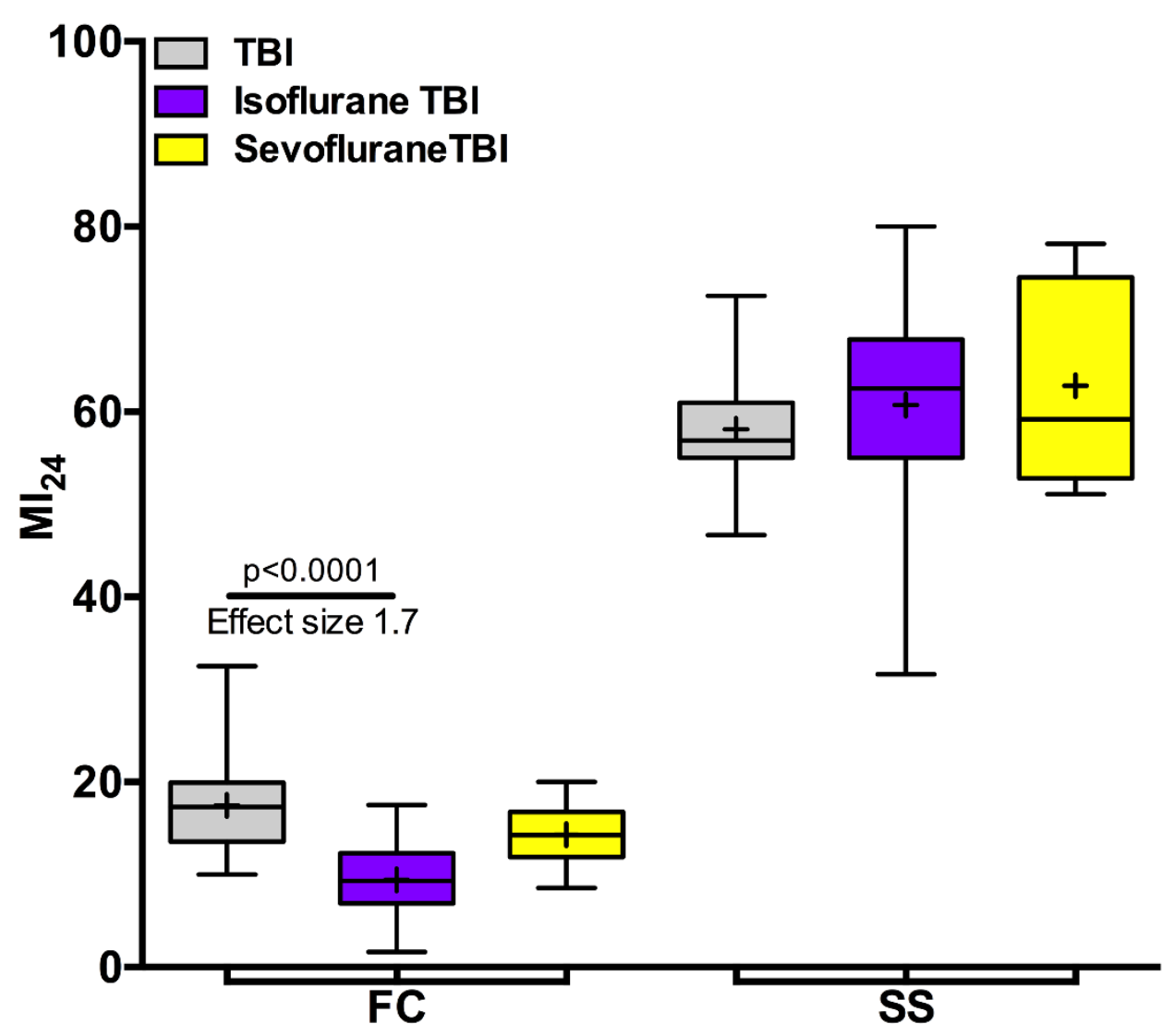

Figure 3. SS flies are resistant to the preconditioning effect of preexposure to volatile general anesthetics (VGAs). Exposure of FC (fed control, i.e., lean flies) to the VGAs to isoflurane prior to TBI (left) reduced the mortality index at $24 \mathrm{~h}\left(\mathrm{MI}_{24}\right)$. The effect was larger with isoflurane than with sevoflurane (Hedge's $g 1.7$ (large) and 0.7 (medium), respectively). For sevoflurane p=0.1. Exposure of SS (starvation-selected, i.e., obese flies) to either VGA did not appreciably affect the $\mathrm{MI}_{24}$ (right). + indicates the mean, the horizontal line indicates the median, the box indicates $25^{\text {th }}$ to $75^{\text {th }}$ percentile and the whiskers extend to the minimal and maximal values.

\subsection{Anesthesia after TBI selectively increases the $\mathrm{MI}_{24}$}

In contrast to the unambiguous effectiveness of preconditioning, the results from exposure to VGAs after ischemia (i.e., postconditioning) have been mixed [22,23]. We tested the effect of exposure to VGAs after TBI using the same doses of isoflurane and sevoflurane as used for preexposure. Exposure of FC flies to isoflurane after TBI increased the 
$\mathrm{MI}_{24}$ from $18.2(3.1, n=16)$ to.25.1 $(6.5, n=8)$, increasing the risk ratio for death to 1.38 [CI 0.81 to $2.38, \mathrm{n}=8$ ) (Fig. 4). In contrast, the $\mathrm{MI}_{24}$ was not appreciably affected by sevoflurane (18.4 (4.3), $n=8)$. These results replicate our findings in $w^{1118}$ flies, in that postexposure with isoflurane but not sevoflurane revealed a toxic potential of VGAs [15,21]. The outcomes differed somewhat in SS flies where exposure to both isoflurane and sevoflurane increased the $\mathrm{MI}_{24}$ from $56.8(8.1, n=26)$ to $73.3(18.4, n=13)$ and to $69.7(17.3, n=13)$, respectively. Postconditioning hence increased the risk ratio for death to 1.28 [CI 1.04 to 1.58], $\mathrm{n}=13$ and 1.23 [CI 0.99 to 1.52 ], $\mathrm{n}=13$ for isoflurane and sevoflurane, respectively. These results in SS flies resemble the increase in $\mathrm{MI}_{24}$ from posttreatment with both agents reported for old $w^{1118}$ flies [21]. We conclude that metabolic changes associated with obesity lower the threshold for VGA toxicity and reveal a toxic potential for sevoflurane in the context of an injured brain.

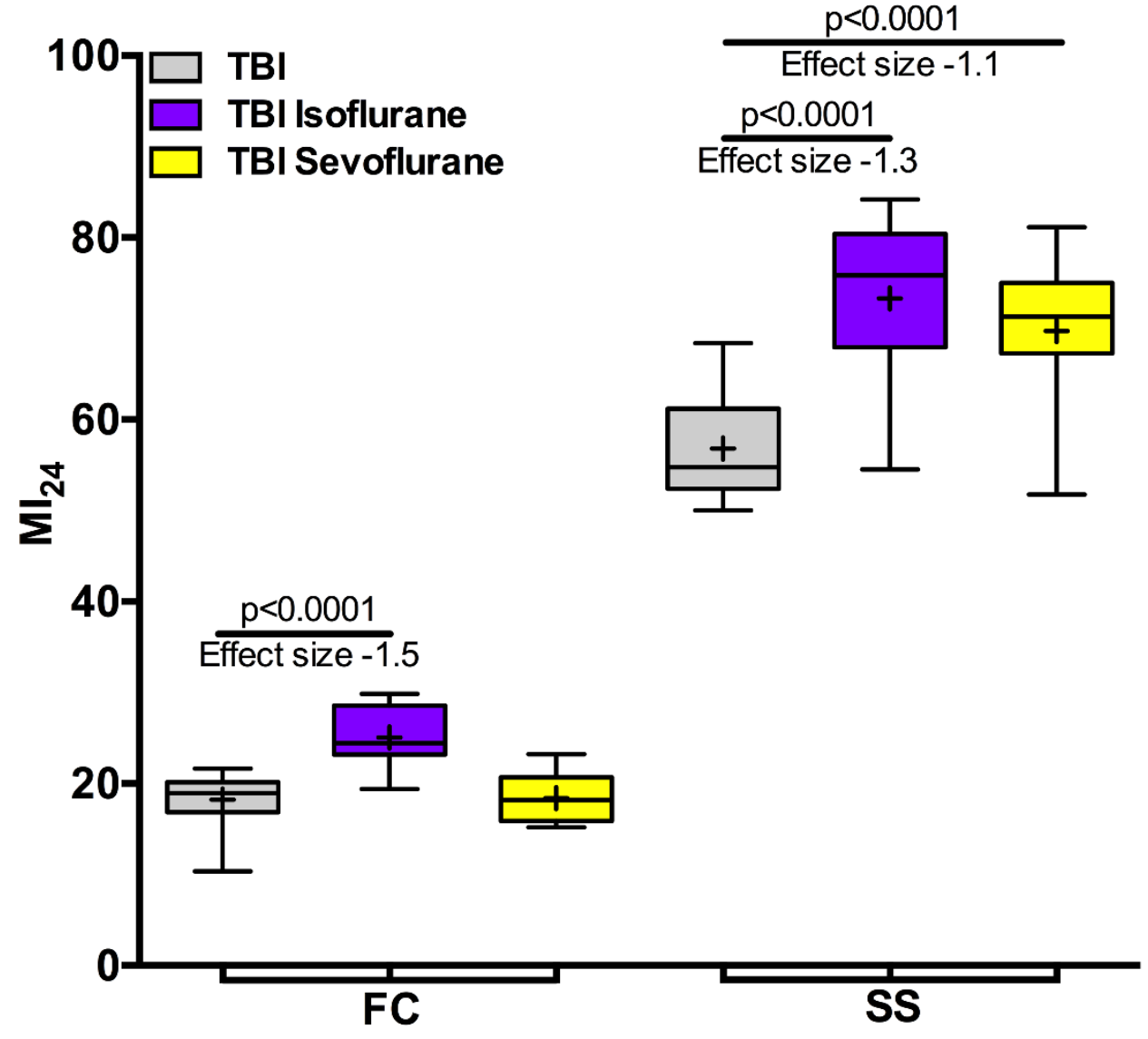

Figure 4. Exposure to volatile general anesthetics (VGAs) after traumatic brain injury (TBI) increases mortality in obese flies. Exposure of FC (fed control, i.e., lean flies) to isoflurane but not to sevoflurane after TBI increased the mortality index at $24 \mathrm{~h}\left(\mathrm{MI}_{24}\right)$ (left). By contrast, exposure of SS (starvation-selected, i.e., obese flies) to isoflurane and sevoflurane increased the $\mathrm{MI}_{24}$ (right). Hedge's $g$ effect size of isoflurane in FC and SS flies was large (-1.5 and -1.3, respectively). The effect of sevoflurane in SS flies was large (-1.1), while there was no effect in FC flies (0). + indicates the mean, the horizontal line indicates the median, the box indicates $25^{\text {th }}$ to $75^{\text {th }}$ percentile and the whiskers extend to the minimal and maximal values.

\subsection{Preexposure and postexposure phenotypes normalize after many generations in the absence of} starvation-selection.

Figures 3 and 4 show that early generation ( $\leq 5^{\text {th }}$ generation) SS flies are distinct from FC flies in their resistance to preconditioning of TBI by isoflurane and sevoflurane and toxicity from postexposure to sevoflurane. To test whether these distinct phenotypes persist after SS flies lose the obese phenotype, we examined flies up to the $10^{\text {th }}$ generation. The $\mathrm{MI}_{24}$ declined proportionally to the loss of weight (Fig. 2, SS-E and SS-L) but remained higher than that of FC flies (Fig. 5). Unexpectedly, after the eighth generation, preexposure 
to isoflurane suppressed the $\mathrm{MI}_{24}$ (Fig. 5a, SS gen 9-10). Concomitantly, postexposure to sevoflurane lost its toxic effect (Fig. 5b, SS gen 9-10). We conclude that pathways mediating the molecular mechanisms of the effects of VGAs on survival after TBI recover after prolonged absence of starvation-selection.

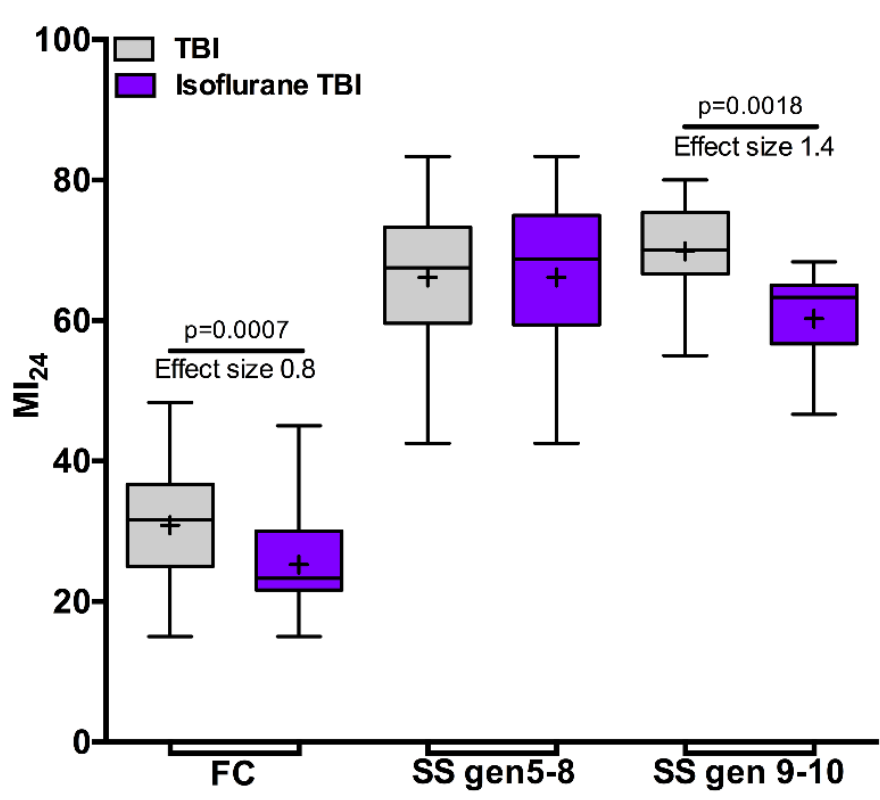

(a)

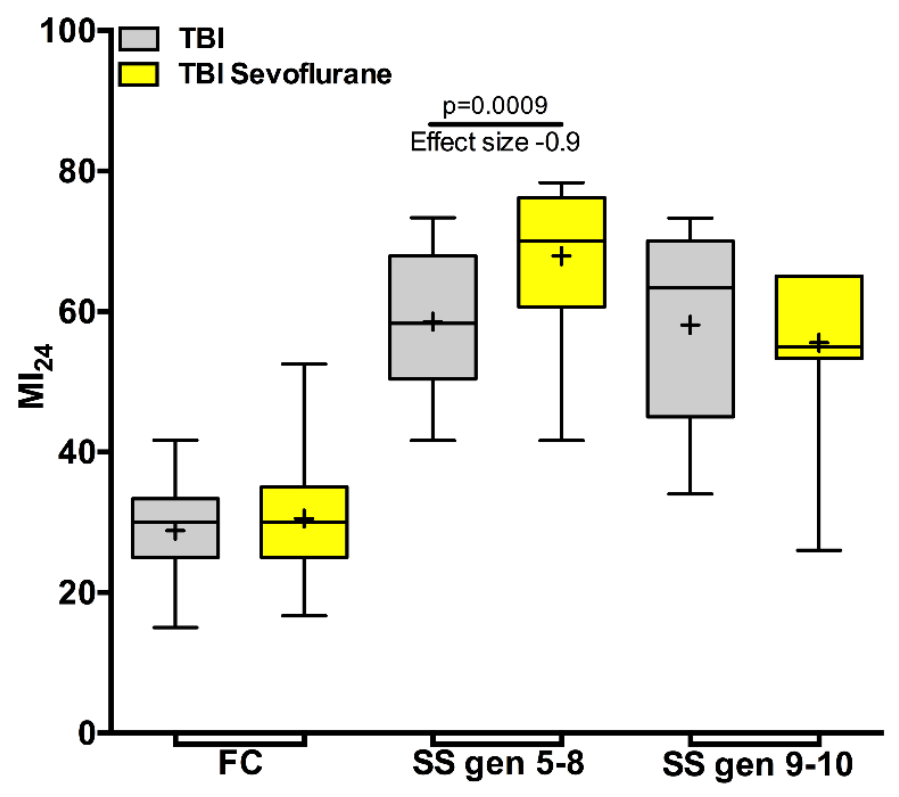

(b)

Figure 5. Anesthetic pharmacodynamics gradually normalize after termination of starvation-selection. (a) SS flies became susceptible to isoflurane preconditioning after the $8^{\text {th }}$ generation. (b) Postexposure toxicity of sevoflurane disappears in SS flies after the $8^{\text {th }}$ generation. Grey bar indicates the $\mathrm{MI}_{24}$ without anesthetic exposure, purple bars indicate preconditioning with $15 \mathrm{~min}$ of $2 \%$ isoflurane, and yellow bars indicate postexposure to $15 \mathrm{~min}$ of $3.5 \%$ sevoflurane. SS gen $5-8$ and SS gen 9-10 indicate the number of generations that the flies reproduced on cornmeal-molasses food. FC are fed control, i.e., lean flies. Note: the $\mathrm{MI}_{24}$ of SS flies remains higher than that of FC flies.

\section{Discussion}

The principal finding of this work is that obesity interferes with preconditioning of the brain (Figs. 3-5). To reach this conclusion, we combined two fly models (TBI and obesity) that reproduce many features of their counterparts in higher animals with the fact that key pharmacokinetic and pharmacodynamic properties of VGAs are evolutionarily conserved [14,15].

Because experimental brain injury and intervention always occur on the background of a brain exposed to anesthetics, inadvertent preconditioning is likely to confound the interpretation of experiments and interventions. Complicating matters, the extent and molecular mechanisms of preconditioning differ among anesthetic agents [10].

Neither the mechanisms of preconditioning nor the causes of its failure are fully understood. Diverse injurious stimuli can precondition the brain but all of them have exceedingly narrow therapeutic indices $[24,25]$. VGAs are exceptional in that preconditioning is induced rapidly but even sustained exposure will not injure the adult 'healthy' brain. Therefore, the mechanism underlying anesthetic preconditioning must differ from other preconditioning stressors that have typically either have a very narrow therapeutic index i.e. a narrow margins between being ineffective, effective and injurious [26] or have to be administered over prolonged periods of time (e.g., hyperthermia [27]). Nevertheless, excessive accumulation of lipids in the heart and associated metabolic abnormalities result in resistance to preconditioning [28].

Numerous not mutually exclusive, mechanisms of anesthetic preconditioning are under investigation. Obesity modulates some of these pathways. For example, the failure of 
preconditioning was attributed to interference with sevoflurane-induced phosphorylation of AMPK (AMP-activated protein kinase) and activation of NOS (nitric oxide synthase) in the myocardium of obese rats [3] and with misregulation of microRNA 21 and NOS by isoflurane in the hearts of diabetic mice [4]. The degree to which similar processes play a role in the brain remains to be investigated, and the experiments presented in this paper are a first step.

The use of Drosophila as a model for clinical conditions is only possible because of extensive evolutionary conservation. For example, over $70 \%$ of human disease-causing genes have orthologues in the fly [29] and, as basic cellular processes are conserved between flies and humans, both share secondary molecular and cellular events triggered by injury [30]. For example, oxidative stress is a major molecular driver of obesity-related complications [31] and plays a similar role in obesity models in flies [32].

Our previous work has shown that pretreatment with VGAs effectively protected flies from death due to TBI [15,21], indicating that some molecular mechanisms by which anesthetics precondition are operational in flies. Here, we expand on our previous findings by showing that, in agreement with data from the rodent myocardium $[3,4]$, obesity interferes with anesthetic preconditioning in brain injury. These findings are particularly relevant for experimental studies of TBI. For example, in TBI induced in rodents either by fluid percussion [33] or controlled-cortical impact [34], diet-induced obesity resulted in worsened outcomes. Both research groups attributed their findings to the effect of diet and/or obesity on biochemical alterations such as brain BDNF (brain derived neurotrophic factor) levels [33]. It is notable though that all animals were exposed to general anesthetics around the time of injury. Therefore, while a role for BDNF is possible, an alternative explanation of differential preconditioning between experimental groups by anesthetics cannot be excluded, illustrating the value of unconventional approaches using invertebrate models to complex, multifactorial pathologies like TBI.

The high $\mathrm{MI}_{24}$ and the lack of preconditioning in SS flies resemble phenotypes of old 'normal' laboratory flies [21]. Aging increases vulnerability to TBI in humans [35] and flies [11] and also reduces the effectiveness of preconditioning in the human myocardium [36]. Because starvation-selection does not shorten lifespan [37], FC and SS flies were injured at the same point in their lifespan. One explanation for our findings may be that obesity associated changes result in a premature-aging-like phenotype revealed under stress caused by TBI. Notably, this phenotype is reversible if the evolutionary pressure of starvation-selection is discontinued: after eight generations, both resistance to isoflurane preconditioning and sevoflurane toxicity are lost, i.e. SS flies revert to the anesthetic phenotype of unselected FC controls (Fig 5).

In summary, while neither TBI nor obesity in flies equals their human counterparts, flies are a useful tool to inform research in higher animals by exploring parameters that would be difficult, expensive or simply unethical to examine in higher animals.

\section{Materials and Methods}

The experiments adhere to applicable ARRIVE (Animal Research: Reporting of In Vivo Experiments) reporting guidelines (preclinical animal research branch of EQUATOR) as far as applicable to flies with the following exceptions: experimental units (vials) were selected arbitrarily and the experimenters was not blinded to treatment as the outcome (death) is unambiguous. We note that the key results were reproduced by two experimenters (JF and HS) working independently at different times in the lab. Approval from the Institutional Animal Care and Use Committee has been waived.

\subsection{Fly Husbandry}

Unless otherwise indicated, experiments were conducted on flies generously provided by Dr. Allen Gibbs (School of Life Sciences, University of Nevada, Las Vegas, NV). The original founding populations for these flies were D. melanogaster collected from Terhune Orchards, Princeton, NJ, USA, in 1999 and maintained as outbred stocks at $25^{\circ} \mathrm{C}$ on 
corn-meal medium. One population underwent starvation-selection (SS population) over multiple generations. Flies used in the experiments described here ranged between generations 120 and 125 of selection. The obese phenotype that developed in the SS population was characterized by increased lipid storage of nearly two times the amount of total lipids as the unselected control population, including a $48 \%$ increase in weight, high fat stores, high whole-body triglyceride levels [16]. SS flies also had a depressed metabolic rate, low activity levels, dilated cardiomyopathy and excess sleep [16-18]. The control population was cultured under the same conditions as the SS population but was provided ad libitum food and water and is referred to as the FC (for fed control) population. Once in our laboratory, all flies were maintained on cornmeal-molasses food at $25^{\circ} \mathrm{C}$ and used at 1-8 days post-eclosion. Once the evolutionary pressure of starvation-selection is removed, SS flies gradually lose the obese phenotype after eight reproductive cycles. Therefore, we used SS flies within 4 reproductive cycles for figures 1, 3.and 4. Fly lines 2P9, D. virilis, and D. funebris were generously provided by Bob Kreber and Dr. Barry Ganetzky (University of Wisconsin-Madison). The $w^{1118}$ line is the standard line maintained in our laboratory. All experiments were conducted on mixed-sex samples. Flies were weighed using an analytical balance with $0.1 \mathrm{mg}$ accuracy (Mettler Toledo XSE104, Columbus, OH).

\subsection{Traumatic brain injury (TBI)}

TBI was induced using a High-Impact Trauma (HIT) device as described previously [11]. All flies were maintained on cornmeal-molasses food at $25^{\circ} \mathrm{C}$. The experimental unit on which analysis is based is a vial that typically contained 20 flies. Experiments that were performed on different days were considered biological replicates. On the day prior to an experiment, eight vials containing 20 mixed-sex SS or FC flies were incubated at $25^{\circ} \mathrm{C}$ with cornmeal-molasses food. The selection of vials was not randomized. On the day of the experiment, flies were rapidly transferred into empty vials. TBI was induced with four strikes from the HIT device with the spring deflected to 90 degrees and 5 min between strikes. Four vials of each phenotype were exposed to VGAs either before or after TBI. The other four vials of each phenotype only underwent TBI. FC and SS flies undergoing anesthesia were tested simultaneously on the Serial Anesthesia Array (SAA). After injury and anesthesia, flies were transferred to vials with cornmeal-molasses food and incubated at $25^{\circ} \mathrm{C}$.

The primary outcome was mortality expressed as the Mortality Index determined 24 $\mathrm{h}$ after TBI $\left(\mathrm{MI}_{24}\right)$. We define the $\mathrm{MI}_{24}$ as the percentage of flies that are dead at $24 \mathrm{~h}$ following TBI minus the percentage of matching uninjured flies that died within the same 24 $\mathrm{h}$ period. Because mortality after TBI does not differ between male and female flies, we performed all experiments on mixed-sex groups. Unless otherwise indicated, at least six independent replicates were performed for each experimental condition. No animals or samples were excluded from the analysis. The experimenter was not blinded to phenotype because SS and FC flies are visibly distinguishable.

\subsection{Anesthesia}

We used a custom-built Serial Anesthesia Array (SAA) to simultaneously expose up to eight samples of 20 flies each to precise doses of VGAs in air, as described previously $[14,15]$. VGAs were administered through the SAA using a Datex-Ohmeda Aestiva/5 anesthesia machine equipped with commercial agent-specific vaporizers (Datex-Ohmeda Inc., Madison, WI). Compressed gas cylinders (Airgas USA, LLC, Radnor, PA) containing air $\left(21 \% \mathrm{O}_{2} / 79 \% \mathrm{~N}_{2}\right)$ provided the carrier gas. To test the effect of obesity on anesthetic pharmacodynamics, we exposed flies to anesthetics either immediately before or after inflicting TBI. We used either $2 \%$ isoflurane or $3.5 \%$ sevoflurane for both exposure protocols. These anesthetic concentrations were behaviorally equivalent and did not affect median and maximum lifespans [14]. The dose of anesthetic administered is reported as concentration (\%) multiplied by duration (hours), e.g., $2 \%$ isoflurane for 2 hours equals $4 \%$ h. All flies resumed movement within less than $1 \mathrm{~h}$ after discontinuing isoflurane or sevoflurane (i.e., no flies died immediately after TBI with or without anesthetic exposure, indicating 
that the doses were safe). A typical assay simultaneously tested two control conditions (iii) and two experimental conditions (iii, iv): (i) no treatment, (ii) anesthesia alone, (iii) TBI alone, and (iv) TBI and anesthesia. All experiments were conducted under normobaric conditions. Mortality under control conditions (i and ii) was less than $1 \%$.

\title{
4.4. Statistical Analysis
}

Data are presented as mean, number of biological replicates $(n)$, [95\% confidence interval]. Each replicate includes 20 individuals except for the determination of weight. To test for significance between treated and untreated FC and SS flies, we used the unpaired two-sample t-test. To compare the $\mathrm{MI}_{24}$ between FC and SS flies subjected to the same treatment, we used the independent two-sample t-test. We quantified the effect of the VGAs on $\mathrm{MI}_{24}$ using relative risk of death, calculated as the relative risk [38]. Data underlying the calculations is plotted in box (interquartile range (IQR) of $\mathrm{P}_{25}$ to $\mathrm{P}_{75}$, i.e., $25^{\text {th }}$ to $75^{\text {th }}$ percentile) and whiskers to maximum and minimum values. We used Prism Graphpad@ for all statistical calculations. We used Hedge's $g$ to calculate effect size for comparing different sample sizes. We used four benchmarks to accommodate the range of our data ( 0 no effect; 0.2 Small effect, cannot be discerned with the naked eye; 0.5 medium effect; and 0.8 large effect, can be seen with the naked eye) (https://www.statisticshowto.com/hedges-g). A minus sign indicates an increase in the $\mathrm{MI}_{24}$.

\begin{abstract}
Author Contributions: Conceptualization, MP and DAW; methodology, JAF and HJS; formal analysis, DJS, JAF, HJS; investigation, ARS and ZPGO; resources, MP and DAW; data curation, DJS; writing - original draft preparation, MP; writing-- review and editing, MP and DW; visualization, DJS; supervision, ZPGO, MP and DAW; project administration, MP; funding acquisition, MP and DAW All authors have read and agreed to the published version of the manuscript.

Funding: This research was funded by a seed grant from the R\&D fund of the Department of Anesthesiology, University of Wisconsin-Madison, by the Clinical and Translational Science Award (CTSA) program, through the NIH National Center for Advancing Translational Sciences (NCATS), grant UL1TR002373, and by the NIH National Institute of Neurological Disorders and Stroke of the National Institutes of Health under Award Number RF1NS114359. The content is solely the responsibility of the authors and does not necessarily represent the official views of the National Institutes of Health.
\end{abstract}

Institutional Review Board Statement: The study was conducted according to the ARRIVE guidelines. Ethical review and approval were waived for this study, due to the study not involving humans or vertebrate animals.

Acknowledgments: The authors thank Allen Gibbs for providing the fly stocks and Mary Roth for editorial assistance.

Conflicts of Interest: The authors declare no conflict of interest. The funders had no role in the design of the study; in the collection, analyses, or interpretation of data; in the writing of the manuscript, or in the decision to publish the results.

\section{References}

1. Kitano, H.; Kirsch, J.R.; Hurn, P.D.; Murphy, S.J. Inhalational anesthetics as neuroprotectants or chemical preconditioning agents in ischemic brain. J Cereb Blood Flow Metab 2007, 27, 1108-1128, doi: 10.1038/sj.jcbfm.9600410.

2. Stadnicka, A.; Marinovic, J.; Ljubkovic, M.; Bienengraeber, M.W.; Bosnjak, Z.J. Volatile anesthetic-induced cardiac preconditioning. J Anesth 2007, 21, 212-219, doi: 10.1007/s00540-006-0486-6.

3. Song, T.; Lv, L.Y.; Xu, J.; Tian, Z.Y.; Cui, W.Y.; Wang, Q.S.; Qu, G.; Shi, X.M. Diet-induced obesity suppresses sevoflurane preconditioning against myocardial ischemia-reperfusion injury: role of AMP-activated protein kinase pathway. Exp Biol Med (Maywood) 2011, 236, 1427-1436, doi: 10.1258/ebm.2011.011165.

4. Ge, Z.D.; Li, Y.; Qiao, S.; Bai, X.; Warltier, D.C.; Kersten, J.R.; Bosnjak, Z.J.; Liang, M. Failure of Isoflurane Cardiac Preconditioning in Obese Type 2 Diabetic Mice Involves Aberrant Regulation of MicroRNA-21, Endothelial Nitric-oxide Synthase, and Mitochondrial Complex I. Anesthesiology 2018, 128, 117-129, doi: 10.1097/ALN.0000000000001926.

5. Szczepaniak, L.S.; Victor, R.G.; Orci, L.; Unger, R.H. Forgotten but not gone: the rediscovery of fatty heart, the most common unrecognized disease in America. Circ Res 2007, 101, 759-767, doi: 10.1161/CIRCRESAHA.107.160457. 
6. Statler, K.D.; Alexander, H.; Vagni, V.; Holubkov, R.; Dixon, C.E.; Clark, R.S.; Jenkins, L.; Kochanek, P.M. Isoflurane exerts neuroprotective actions at or near the time of severe traumatic brain injury. Brain Res 2006, 1076, 216-224, doi: 10.1016/j.brainres.2005.12.106.

7. Tétrault, S.; Chever, O.; Sik, A.; Amzica, F. Opening of the blood-brain barrier during isoflurane anaesthesia. Eur J Neurosci 2008, 28, 1330-1341, doi: 10.1111/j.1460-9568.2008.06443.x.

8. Staib-Lasarzik, I.; Kriege, O.; Timaru-Kast, R.; Pieter, D.; Werner, C.; Engelhard, K.; Thal, S.C. Anesthesia for euthanasia influences mRNA expression in healthy mice and after traumatic brain injury. J Neurotrauma 2014, 31, 1664-1671, doi: 10.1089/neu.2013.3243.

9. Semple, B.D.; Sadjadi, R.; Carlson, J.; Chen, Y.; Xu, D.; Ferriero, D.M.; Noble-Haeusslein, L.J. Long-Term Anesthetic-Dependent Hypoactivity after Repetitive Mild Traumatic Brain Injuries in Adolescent Mice. Dev Neurosci 2016, 38, 220-238, doi: $10.1159 / 000448089$.

10. Statler, K.D.; Alexander, H.; Vagni, V.; Dixon, C.E.; Clark, R.S.; Jenkins, L.; Kochanek, P.M. Comparison of seven anesthetic agents on outcome after experimental traumatic brain injury in adult, male rats. J Neurotrauma 2006, 23, 97-108, doi: 10.1089/neu.2006.23.97.

11. Katzenberger, R.J.; Loewen, C.A.; Wassarman, D.R.; Petersen, A.J.; Ganetzky, B.; Wassarman, D.A. A Drosophila model of closed head traumatic brain injury. Proc Natl Acad Sci U S A 2013, 110, E4152-4159, doi: 10.1073/pnas.1316895110.

12. Saikumar, J.; Byrns, C.N.; Hemphill, M.; Meaney, D.F.; Bonini, N.M. Dynamic neural and glial responses of a head-specific model for traumatic brain injury in Drosophila. Proc Natl Acad Sci U S A 2020, 117, 17269-17277, doi: 10.1073/pnas.2003909117.

13. Putnam, L.J.; Willes, A.M.; Kalata, B.E.; Disher, N.D.; Brusich, D.J. Expansion of a fly TBI model to four levels of injury severity reveals synergistic effects of repetitive injury for moderate injury conditions. Fly (Austin) 2019, 13, 1-11, doi: 10.1080/19336934.2019.1664363.

14. Olufs, Z.P.G.; Loewen, C.A.; Ganetzky, B.; Wassarman, D.A.; Perouansky, M. Genetic variability affects absolute and relative potencies and kinetics of the anesthetics isoflurane and sevoflurane in Drosophila melanogaster. Sci Rep 2018, 8, 2348, doi: 10.1038/s41598-018-20720-7.

15. Fischer, J.A.; Olufs, Z.P.G.; Katzenberger, R.J.; Wassarman, D.A.; Perouansky, M. Anesthetics Influence Mortality in a Drosophila Model of Blunt Trauma With Traumatic Brain Injury. Anesth Analg 2018, 126, 1979-1986, doi: 10.1213/ANE.0000000000002906.

16. Reynolds, L.A. The Effects of Starvation Selection on Drosophila melanogaster Life History and Development. Ph.D. dissertation, University of Nevada, Las Vegas, NV, 2013.

17. Masek, P.; Reynolds, L.A.; Bollinger, W.L.; Moody, C.; Mehta, A.; Murakami, K.; Yoshizawa, M.; Gibbs, A.G.; Keene, A.C. Altered regulation of sleep and feeding contributes to starvation resistance in Drosophila melanogaster. J Exp Biol 2014, 217, 31223132, doi: 10.1242/jeb.103309.

18. Hardy, C.M.; Birse, R.T.; Wolf, M.J.; Yu, L.; Bodmer, R.; Gibbs, A.G. Obesity-associated cardiac dysfunction in starvation-selected Drosophila melanogaster. Am J Physiol Regul Integr Comp Physiol 2015, 309, R658-667, doi: 10.1152/ajpregu.00160.2015.

19. Katzenberger, R.J.; Chtarbanova, S.; Rimkus, S.A.; Fischer, J.A.; Kaur, G.; Seppala, J.M.; Swanson, L.C.; Zajac, J.E.; Ganetzky, B.; Wassarman, D.A. Death following traumatic brain injury in Drosophila is associated with intestinal barrier dysfunction. Elife 2015, 4, 1-24, doi: 10.7554/eLife.04790.

20. Unckless, R.L.; Rottschaefer, S.M.; Lazzaro, B.P. A genome-wide association study for nutritional indices in Drosophila. G3 (Bethesda) 2015, 5, 417-425, doi: 10.1534/g3.114.016477.

21. Schiffman, H.J.; Olufs, Z.P.G.; Lasarev, M.R.; Wassarman, D.A.; Perouansky, M. Ageing and genetic background influence anaesthetic effects in a D. melanogaster model of blunt trauma with brain injury(dagger). Br J Anaesth 2020, 125, 77-86, doi: 10.1016/j.bja.2020.03.029.

22. Wright, N.E.; Strong, J.A.; Gilbart, E.R.; Shollenbarger, S.G.; Lisdahl, K.M. 5-HTTLPR Genotype Moderates the Effects of Past Ecstasy Use on Verbal Memory Performance in Adolescent and Emerging Adults: A Pilot Study. PLoS One 2015, 10, e0134708, doi: 10.1371/journal.pone.0134708.

23. Li, L.; Zuo, Z. Isoflurane postconditioning induces neuroprotection via Akt activation and attenuation of increased mitochondrial membrane permeability. Neuroscience 2011, 199, 44-50, doi: 10.1016/j.neuroscience.2011.10.022.

24. Lucchinetti, E.; da Silva, R.; Pasch, T.; Schaub, M.C.; Zaugg, M. Anaesthetic preconditioning but not postconditioning prevents early activation of the deleterious cardiac remodelling programme: evidence of opposing genomic responses in cardioprotection by pre- and postconditioning. Br J Anaesth 2005, 95, 140-152, doi: 10.1093/bja/aei155.

25. Gidday, J.M. Cerebral preconditioning and ischaemic tolerance. Nat Rev Neurosci 2006, 7, 437-448, doi: $10.1038 / \mathrm{nrn} 1927$.

26. Obrenovitch, T.P. Molecular physiology of preconditioning-induced brain tolerance to ischemia. Physiol Rev 2008, 88, 211-247, doi: 10.1152/physrev.00039.2006.

27. Shohami, E.; Novikov, M.; Horowitz, M. Long term exposure to heat reduces edema formation after closed head injury in the rat. Acta Neurochir Suppl (Wien) 1994, 60, 443-445, doi: 10.1007/978-3-7091-9334-1_121.

28. Nakanishi, T.; Kato, S. Impact of diabetes mellitus on myocardial lipid deposition: an autopsy study. Pathol Res Pract 2014, 210, 1018-1025, doi: 10.1016/j.prp.2014.04.008.

29. Reiter, L.T.; Potocki, L.; Chien, S.; Gribskov, M.; Bier, E. A systematic analysis of human disease-associated gene sequences in Drosophila melanogaster. Genome Res 2001, 11, 1114-1125, doi: 10.1101/gr.169101.

30. Chow, C.Y.; Reiter, L.T. Etiology of Human Genetic Disease on the Fly. Trends Genet 2017, 33, 391-398, doi: 10.1016/j.tig.2017.03.007. 
31. Furukawa, S.; Fujita, T.; Shimabukuro, M.; Iwaki, M.; Yamada, Y.; Nakajima, Y.; Nakayama, O.; Makishima, M.; Matsuda, M.; Shimomura, I. Increased oxidative stress in obesity and its impact on metabolic syndrome. J Clin Invest 2004, 114, 1752-1761, doi: 10.1172/JCI21625.

32. Trindade de Paula, M.; Poetini Silva, M.R.; Machado Araujo, S.; Cardoso Bortolotto, V.; Barreto Meichtry, L.; Zemolin, A.P.; Wallau, G.L.; Jesse, C.R.; Franco, J.L.; Posser, T., et al. High-Fat Diet Induces Oxidative Stress and MPK2 and HSP83 Gene Expression in Drosophila melanogaster. Oxid Med Cell Longev 2016, 2016, 4018157, doi: 10.1155/2016/4018157.

33. Wu, A.; Molteni, R.; Ying, Z.; Gomez-Pinilla, F. A saturated-fat diet aggravates the outcome of traumatic brain injury on hippocampal plasticity and cognitive function by reducing brain-derived neurotrophic factor. Neuroscience 2003, 119, 365-375, doi: 10.1016/s0306-4522(03)00154-4.

34. Hoane, M.R.; Swan, A.A.; Heck, S.E. The effects of a high-fat sucrose diet on functional outcome following cortical contusion injury in the rat. Behav Brain Res 2011, 223, 119-124, doi: 10.1016/j.bbr.2011.04.028.

35. Maas, A.I.; Stocchetti, N.; Bullock, R. Moderate and severe traumatic brain injury in adults. Lancet Neurol 2008, 7, 728-741, doi: 10.1016/S1474-4422(08)70164-9.

36. Mio, Y.; Bienengraeber, M.W.; Marinovic, J.; Gutterman, D.D.; Rakic, M.; Bosnjak, Z.J.; Stadnicka, A. Age-related attenuation of isoflurane preconditioning in human atrial cardiomyocytes: roles for mitochondrial respiration and sarcolemmal adenosine triphosphate-sensitive potassium channel activity. Anesthesiology 2008, 108, 612-620, doi: 10.1097/ALN.0b013e318167af2d.

37. Archer, M.A.; Phelan, J.P.; Beckman, K.A.; Rose, M.R. Breakdown in correlations during laboratory evolution. II. Selection on stress resistance in Drosophila populations. Evolution 2003, 57, 536-543, doi: 10.1111/j.0014-3820.2003.tb01545.x.

38. Altman, D.G. Practical Statistics for Medical Research, 1st ed.; Chapman and Hall/CRC: London, 1991. 